\title{
The DISINFECT Initiative: Decreasing the Incidence of Surgical INFECTions in Gynecologic Oncology
}

\author{
Jolyn S. Taylor, MD MPH ${ }^{1}$, Claire A. Marten, PharmD², Mark F. Munsell, MS ${ }^{3}$, Charlotte C. \\ Sun, DrPh ${ }^{1}$, Kimberly A. Potts, RN ${ }^{4}$, Jennifer K. Burzawa, MD ${ }^{1}$, Alpa M. Nick, MD MS ${ }^{1}$, \\ Larissa A. Meyer, MD MPH ${ }^{1}$, Keith Myers, BS ${ }^{1}$, Diane C. Bodurka, MD MPH ${ }^{5}$, Thomas A. \\ Aloia, $\mathbf{M D}^{6}$, Charles F. Levenback, $\mathbf{M D}^{1}$, David R Lairson, $\mathbf{P h D}^{7}$, and Kathleen $\mathbf{M}$. Schmeler, \\ MD 1 \\ ${ }^{1}$ The University of Texas MD Anderson Cancer Center, Department of Gynecologic Oncology and \\ Reproductive Medicine \\ ${ }^{2}$ The University of Texas MD Anderson Cancer Center, Division of Pharmacy \\ ${ }^{3}$ The University of Texas MD Anderson Cancer Center, Department of Biostatistics \\ ${ }^{4}$ The University of Texas MD Anderson Cancer Center, Department of Perioperative Services \\ ${ }^{5}$ The University of Texas MD Anderson Cancer Center, Department of Clinical Education \\ ${ }^{6}$ The University of Texas MD Anderson Cancer Center, Department of Surgical Oncology \\ ${ }^{7}$ The University of Texas School of Public Health, Department of Management \& Policy Sciences
}

\section{Abstract}

Background-Surgical site infections (SSI) lead to increased patient morbidity and healthcare costs. Our objective was to decrease the SSI rate following gynecologic surgery.

Methods-Adult patients undergoing abdominal surgery for gynecologic malignancy or benign disease received the following: patient education; preoperative antibacterial soap; appropriate antibiotic prophylaxis; change of gloves and use of clean instruments at surgical closure; surgical dressing for 48 hours; and a post-discharge phone call. The baseline SSI rate was determined retrospectively (4/1/14-6/30/14) while the post-intervention SSI rate was determined prospectively (2/16/15-10/15/15). The main outcome was the overall SSI rate with secondary outcomes including the rate of superficial, deep, incisional and organ space infection as well as the bundle's cost-effectiveness.

Results-A total of 232 baseline and 555 post-intervention patients were included. There were no differences between baseline and post-intervention groups with regard to median BMI, surgical approach, receipt of preoperative chemotherapy and/or radiation therapy and cases including

Corresponding Author: Kathleen M. Schmeler, Department of Gynecologic Oncology and Reproductive Medicine, The University of Texas MD Anderson Cancer Center, Office: (713) 563-4578, Fax: (713) 745-2398, KSchmele@ mdanderson.org, Cancer Prevention Building, Suite CPB6.3584, 1155 Pressler Street, Unit 1362 Houston, Texas 77030.

Conflict of Interest: The following authors report conflicts of interest: Larissa A. Meyer, MD (Honaria: TRM Oncology; Research Funding: AstraZeneca) and Kathleen M Schemler, MD (Research Funding: Cepheid; Patents, Royalties or Other Intellectual Property: UpToDate). All remaining authors report no conflicts of interest. 
bowel surgery. Overall SSI rate decreased significantly from baseline (12.5\%) to post-intervention (7.4\%) (OR 0.56 [0.37-0.85]; $\mathrm{p}=0.01)$. A $40 \%$ decrease was noted in the rate of superficial and deep infections ( $9.5 \%$ vs. $5.9 \%$; OR 0.60 [0.38-0.97]; $\mathrm{p}=0.04)$ and SSI after open surgery $(21.4 \%$ vs. $13.2 \%$; OR 0.56 [0.34-0.92]; $\mathrm{p}=0.03$ ). The estimated cost of the intervention was $\$ 19.26 /$ case and the net total amount saved during the post-intervention period was $\$ 65,625 / \mathrm{month}$.

Conclusions-This bundled intervention led to a significant decrease in the overall SSI rate and was cost-effective. The largest decreases in SSI were in incisional infections and following open surgery.

\section{Introduction}

Surgical site infections (SSI) occur within the surgical skin incision or internally within the body following surgery. Development of SSI adversely affects health outcomes and is associated with increased morbidity and mortality among cancer patients. ${ }^{1-3}$ SSI are also associated with increased medical costs, estimated to be up to $\$ 1.6$ billion annually. ${ }^{2-4}$ The rate of SSI is reported by national agencies as a marker of the safety and quality of healthcare provided by an institution. The rate of SSI following surgery for gynecologic malignancy has been estimated to be 10 to $15 \% .^{1}$

Given that the development of SSI represents a substantial quality of care concern for the healthcare system, many quality improvement initiatives have attempted to address this issue $^{5-12}$ There are varying levels of evidence to support individual interventions implemented during the pre-operative, intra-operative and post-operative time periods. As a result, many institutions implement a 'bundle' of interventions aimed at decreasing the rate of SSI rather than a single intervention. ${ }^{13-15}$ To date, there is limited evidence regarding the effect of bundled initiatives on SSI rates following surgery for gynecologic malignancy. ${ }^{15,16}$ Furthermore, the cost-effectiveness analyses of such initiatives have not been reported. The objective of this report is to describe the results and cost-effectiveness of a quality improvement initiative to reduce the rate of SSI within the Department of Gynecologic Oncology and Reproductive Medicine at a tertiary academic center specializing in cancer care.

\section{Methods}

\section{Study Design}

Following Quality Improvement Assessment Board (QIAB) approval, which functions as the Institutional Review Board for quality improvement research, we performed a retrospective review of all abdominal surgery cases completed within the Department of Gynecologic Oncology and Reproductive Medicine at the University of Texas MD Anderson Cancer Center from 4/1/14-6/30/14 to establish baseline SSI data. The length of time for the baseline and post-intervention periods were determined from a power analysis assuming a baseline rate of $15 \%$, with $80 \%$ power to detect a $50 \%$ reduction in SSI rate using a onesided $a=0.05$. SSI was defined as an infection of the surgical incision or organ space requiring antibiotics within 30 days of surgery. This definition was chosen instead of the more restrictive definitions used by reporting agencies as we wished to include all SSI 
cases. ${ }^{17,18}$ Receipt of antibiotics to treat an infection is an objective measure with clinical impact on the patient. Data for the post-intervention period (2/16/15-10/15/15) were captured prospectively following implementation of the intervention bundle for all abdominal surgery cases performed within our department.

The types of SSI described in this manuscript were adapted from the definitions endorsed by the Centers for Disease Control and Prevention: superficial SSI involves the skin to the level above the fascia; deep SSI involves the fascia and/or muscle layer; and organ space infections (OSI) involve any part of the body deeper than the fascial/muscle layers that is opened or manipulated during the operative procedure. ${ }^{19}$ Given the similarities in the pathophysiology of preventing and developing superficial and deep SSI, as well as the possible ambiguity of differentiating these types of SSI based on available documentation, these two SSI categories were also combined and defined as incisional SSI. Superficial or deep SSI could be present with OSI. Cases performed jointly with other surgical services were excluded.

The direct costs of the intervention were determined from the hospital dispensary. The cost of SSI was determined from the hospital perspective using data from the Department of Clinical Revenue and Reimbursement. As Medicare does not reimburse for readmissions due to SSI within 30 days of surgery, the cost of readmission due to SSI is a financial loss to the hospital. The paid amounts received from private insurance carriers who provided reimbursement for hospital readmissions related to treatment of SSI within the 30 day postoperative period were used to create an average value for the cost of each type of SSI. When comparing costs between the baseline and post-intervention periods, the three month baseline period was extrapolated to be equal to the eight month post-intervention period. The cost to the hospital during the post-intervention period included the cost of the intervention bundle as well as the estimated cost related to SSI readmission. The unit of effectiveness was defined as the change in the overall SSI rate between baseline and post-intervention periods.

\section{Intervention Bundle}

A bundled intervention was implemented for all abdominal surgeries beginning $2 / 16 / 15$. The intervention bundle included evidence-based measures adapted to our institutional practices. The pre-operative interventions included showering with antibacterial soap the night before and morning of surgery and receiving appropriate prophylactic antibiotics with appropriate timing and dose before surgical incision. ${ }^{12-14,20,21}$ Pre-operative antibiotics were standardized based on the 2013 joint antimicrobial prophylaxis guidelines by the American Society of Health-System Pharmacists (ASHP), the Infectious Diseases Society of America (IDSA), the Surgical Infection Society (SIS), and the Society for Healthcare Epidemiology of America (SHEA). ${ }^{22}$ Antibiotic options included cefazolin or cefoxitin (preferred option for bowel surgery) or combination clindamycin and ciprofloxacin for penicillin allergic patients. ${ }^{22}$ Intra-operative interventions included appropriately re-dosing antibiotics, using separate sterile instruments, suction tip, electrocautery device and gloves for fascial and skin closure and changing gowns if vaginal or perineal contamination. ${ }^{13,14,20}$ Finally, the postoperative interventions included labeling the surgical dressing in the operating room with the date and time for removal on the second post-operative day (24-48 hours post-operatively), 
providing patient education handouts regarding signs and symptoms of SSI and giving a follow up phone call to the patient 48-72 hours after surgery with further SSI education and assessment. ${ }^{11,13,14}$

\section{Statistical Analysis}

Patient demographics, clinical characteristics and SSI rates were compared between baseline and post-intervention groups using Fisher's exact and Mann-Whitney tests. Categorical and continuous variables were described using proportions and measures of central tendency, respectively. Multivariate exact logistic regression analyses of the SSI outcomes were performed with inclusion of all covariates significant to less than $\mathrm{p}=0.25$ on univariate analysis. Backward elimination was then performed until only covariates significant to $p<0.05$ remained. The main outcome was the overall rate of SSI with stratification by implementation period of the intervention bundle. Secondary outcomes included the rate of superficial, deep, incisional and OSI as well as the cost-effectiveness of the bundled intervention. OSI could be present concurrently with either superficial or deep infections. Variables assessed for inclusion in the multivariate analysis included use of the intervention bundle, surgical approach, current smoking status, receipt of appropriate prophylactic antibiotics, appropriate redosing of antibiotics, body mass index (BMI) (stratified as $<25$, 25-30, >30), race (white vs. non-white), age, estimated blood loss (EBL), having a Charlson Comorbidity Index Score $>1$, diabetes, receipt of preoperative chemotherapy, receipt of preoperative radiation, surgical time and undergoing bowel surgery. All covariates were binary (yes/no) except for age, EBL, BMI and surgical time. Model diagnostics were assessed and no major assumptions were violated. $\mathrm{P} \leq 0.05$ indicated statistical significance for all comparisons and analyses of primary and secondary outcomes. Statistical assessment was two-sided when comparing demographic characteristics and one-sided for SSI outcomes. Stata ${ }^{\circledR}$ version 13.1 statistical software was used for all statistical analyses (College Station, TX).

\section{Results}

Two hundred and thirty-two baseline surgical cases were compared to 555 post-intervention surgical cases. Patient demographics for the baseline and post-intervention groups are shown in Table 1. There were no significant differences between the groups with regards to median BMI (28.9 vs. $29.4 \mathrm{~kg} / \mathrm{m}^{2}, \mathrm{p}=0.30$ ), surgical approach (laparotomy $48 \%$ vs. $41 \%, \mathrm{p}=0.06$ ), receipt of preoperative chemotherapy $(17 \%$ vs. $14 \%, \mathrm{p}=0.18)$ or radiation therapy $(2 \%$ vs. $1 \%, \mathrm{p}=0.54)$, and bowel surgery ( $23 \%$ vs. $21 \%, \mathrm{p}=0.39$ ). Compliance with the bundled intervention was tracked by survey and chart review by members of the quality improvement team. Compliance with the intervention bundle, accept for receipt of appropriate preoperative antibiotics, ranged from $84-94 \%$ throughout the study period. The initial compliance with appropriate receipt of pre-operative antibiotic was $62 \%$, however, this increased by the end of the study period to $74 \%$.

As shown in Figure 1, implementation of the intervention bundle correlated with a decrease in the overall SSI rate from baseline (12.5\%) to post-intervention (7.4\%) (OR 0.56 [0.37$0.85] ; p=0.01$ ). Among the types of SSI, incisional infections decreased by the largest 
amount from baseline (9.5\%) to post-intervention (5.9\%) (OR 0.60 [0.38-0.97]; $\mathrm{p}=0.04$ ) and was the only type to reach statistical significance. However, decreases were seen in all types of SSI including superficial (7.8\% vs. 5.2\%; OR 0.66 [0.39-1.09]; $\mathrm{p}=0.09$ ), deep (1.7\% vs. $0.7 \%$; OR 0.41 [0.13-1.33]; $\mathrm{p}=0.11$ ) and OSI (3.9\% vs. $2.3 \%$; OR 0.59 [0.29-1.23]; $\mathrm{p}=0.12$ ). The baseline and post-intervention rates of SSI by type of SSI are shown in Table 2. In addition, the rate of SSI decreased by $40 \%$ following laparotomy $(21.4 \%$ v $13.2 \%$; OR $0.56[0.34-0.92] ; \mathrm{p}=0.03)$ but was unchanged following minimally invasive surgery $(4.2 \% \mathrm{v}$ $3.4 \%$; OR 0.80 [0.32-1.97]; $\mathrm{p}=0.34$ ).

On multivariate analysis, the intervention bundle remained significantly correlated with a decreased overall likelihood of developing SSI (OR 0.64 [0.41-0.98]; $\mathrm{p}=0.04$ ) (Table 3). Appropriate re-dosing of intraoperative antibiotics was also found to be protective against developing an SSI on multivariate analysis (OR 0.45 [0.24-0.87]; p=0.02). Factors associated with an increase in the rate of SSI on multivariate analysis included: laparotomy (OR 4.90 [3.01-7.98]; $\mathrm{p}<0.001$ ) and current smoking (OR 1.95 [1.03-3.69]; $\mathrm{p}=0.04$ ).

The cost of the bundled intervention was estimated to be $\$ 1,336.25$ a month or $\$ 19.26$ per case (Table 4). This included the one-time cost of purchasing additional surgical trays for the closure of the skin and fascia. Personnel cost were not included in the analysis as all team members assisted with the initiative as part of their regular employment. However, a sensitivity analysis was performed which included theoretical personnel cost.

The cost of each type of SSI from the hospital perspective is shown in Table 2. For the costeffectiveness analysis, the types of SSI were considered to be mutually exclusive. If a patient developed a superficial or deep and OSI infection, it was calculated as only an OSI so as to not overestimate the amount saved. In addition, the overall amount saved only included the values for the superficial, deep and OSI infections as the values for the incisional SSI are included within the superficial and deep SSI values. The estimated amount saved through avoidance of SSI during the study period was $\$ 535,686$ or $\$ 66,961$ per month. After factoring in the cost of the intervention bundle, the amount saved is $\$ 524,996$ or $\$ 65,625$ per month. The intervention group was found to be more effective (lower rate of SSI) and less expensive than the non-intervention group.

Three sensitivity analyses were performed in order to test the robustness of the costeffectiveness analyses. First, the efficacy of the bundled intervention was reduced by $50 \%$. In this scenario, the amount saved through avoidance of SSI was $\$ 262,877$ or $\$ 32,859$ a month and the intervention was still cost-saving and resulted in net-savings of $\$ 252,187$ or $\$ 31,523$ a month. Second, the cost to the hospital of SSI was reduced by $75 \%$. Even with a $75 \%$ decrease in the cost of readmission to treat SSI, the intervention remained cost-saving with a net-savings of $\$ 123,232$ or $\$ 15,404$ a month. Lastly, the cost of the intervention bundle was increased by adding personnel costs equivalent to the standard yearly salary of a research data coordinator at this institution of $\$ 37,000 .{ }^{23}$ In this scenario, the cost of the intervention bundle was estimated to be $\$ 47,619$ or averaged to be $\$ 85.80$ per case. The intervention remained net cost-saving with an overall reduction in cost of $\$ 488,067$ or $\$ 61,008$ a month. Finally, a worst-case scenario was performed in which the efficacy was reduced by $50 \%$, the cost of SSI readmission was reduced by $75 \%$ and the cost of the 
intervention was increased by additional personnel costs. In this scenario the intervention still resulted in a net savings of $\$ 19,342$ or $\$ 2,418$ a month. In all of these scenarios, the intervention group remained dominant and was cost-effective compared to the nonintervention group.

\section{Discussion}

SSIs are potentially avoidable post-operative complications. Implementation of the described bundled intervention significantly decreased the overall rate of SSI from $12.5 \%$ to $7.4 \%$. The largest improvements were seen in the rate of incisional SSI and the rate of SSI following laparotomy. Our results are consistent with results reported by other initiatives. Duke University and the Mayo Clinic have implemented bundled interventions following colorectal and gynecologic surgery with decreases in SSI rates from 50 to $80 \% .^{13,14,16}$ Among high-risk gynecologic oncology patients, Novetsky et al. implemented a five-part bundled intervention with reduction in the rate of SSI by $60 \% .{ }^{15}$

The intervention was also cost-effective. We estimate that it could reduce hospital costs by over $\$ 65,000$ a month. While previous studies have addressed reducing the morbidity associated with SSI, the literature evaluating the cost-effectiveness of SSI reduction interventions is limited. Previous studies have described the cost-effectiveness of single interventions but not the effect of implementing a bundled intervention. ${ }^{24,25}$ Other studies have described the estimated costs associated with certain types of infections, but not a full cost assessment of implementing a bundled intervention. ${ }^{13}$ While it is important to decrease patient morbidity through reduction in the SSI rate, it is also pertinent to assess and analyze the cost-effectiveness of these quality improvement initiatives. Future informed decisions to optimize the delivery of high-quality healthcare will depend on the efficacy of different interventions, and their cost-effectiveness.

A limitation of this analysis was that detection of SSI was dependent upon adequate documentation in the clinical record and could be subjective. Furthermore, as there was concurrent education of the clinical care teams regarding SSI related to this initiative, it was possible that the superficial SSI rate declined, in part, due to more restrictive antibiotic dispensing and clearer documentation. However, all sub-types of SSI decreased in rate between baseline and post-intervention periods, even the less subjective sub-types of deep and OSI. This benefit was not likely due to reporting bias. This analysis could only assess the effect of the bundled intervention as a whole and the contributions of the individual components of the intervention could not be evaluated. Finally, this initiative was implemented at a large academic cancer center and the results, especially the cost analysis, may not be applicable to all settings as insurance reimbursements vary significantly between healthcare locations.

The strengths of this initiative include the prospective review of all surgical cases in order to provide frequent, relevant feedback to the clinical care teams. Other published quality improvement initiatives have relied on national agencies which can lag months behind in reporting results and have varying inclusion criteria for review of surgical cases. ${ }^{13,14,17,18}$ In addition, by including all abdominal surgery cases, our results are applicable to all patients 
undergoing surgery for gynecologic malignancy or benign gynecologic disease. This is in contrast to other studies which evaluated only certain high-risk groups. ${ }^{15,26,27}$

In conclusion, this quality improvement initiative utilizing a bundled intervention reduced the overall rate of SSI following surgery for gynecologic malignancy. The initiative was cost-effective and led to substantial reductions in hospital costs. Reduction of SSI is a highimpact goal leading to improvement in the quality of patient care and reduction in healthcare costs. Additional study is needed to define the optimal components of the intervention bundle and to confirm sustainability.

\section{Acknowledgments}

The authors would like to acknowledge the following people for their contributions to this initiative: Katherine E. Cain, PharmD; Lynn M. Cloutier, RN, MSN, ACNP-BC; Shauna L. Fenton, RN, MSN; Linda S. Graviss, MT, (ASCP),CIC; Cheryl A. Hubbs, RN; Deepthi A. James, MSN, RN, FNP-C; Johnrich R. Levine, MSN, MHA, MPA, BSN, RN, PNE-C; Keith N. Myers, BS; Anne K. Park, MS, MPH; Deandra Sandles, RN, MSN, CNOR; Tara N. Tatum, RN, MBA; Shital Vachhani, MD; Kathy M. Ware, RN

Financial support: This research was supported in part by the National Institutes of Health through MD Anderson Cancer Center's Support Grant CA016672. Dr. Taylor's work on this project was supported by a NIH T32 grant, Training of Academic Gynecologic Oncologists, from the National Cancer Institute (5T32-CA101642). The funding source had no direct role in preparing or reviewing this study nor this manuscript.

\section{References}

1. Tran CW, McGree ME, Weaver AL, et al. Surgical site infection after primary surgery for epithelial ovarian cancer: Predictors and impact on survival. Gynecologic Oncology. 2015; 136(2):278-284. [PubMed: 25499962]

2. Kirkland KB,JP, Trivette SL, Wilkinson WE, Sexton DJ. The impact of surgical-site infections in the 1990s: attributable mortality, excess length of hospitalization, and extra costs. Infect Control Hosp Epidemiol. 1999; 20:725-730. [PubMed: 10580621]

3. Itani KF. Care bundles and prevention of surgical site infection in colorectal surgery. JAMA. 2015; 314(3):289-290. [PubMed: 26197189]

4. de Lissovoy GF,K, Hutchins V, Murphy D, Song D, Vaughn BB. Surgical site infection: incidence and impact on hospital utilization and treatment costs. American Journal of Infection Control. 2009; 37(5):387-397. [PubMed: 19398246]

5. Alexander JF, Boyajian M, Palmquist J, Morris MJ. The influence of hair-removal methods on wound infections. Archives of Surgery. 1983; 118(3):347-352. [PubMed: 6824435]

6. Bullock, RvD, JR., Ketelbey, W., Reinach, SG. A double-blind placebocontrolled trial of perioperative prophylactic antibiotics for elective neurosurgery. Journal of Neurosurgery. 1988; 69(5):687-691. [PubMed: 3054011]

7. Page CB,JM, Fletcher JR, McManus AT, Solomkin JS, Wittmann DH. Antimicrobial prophylaxis for surgical wounds. Guidelines for clinical care. Archives of Surgery. 1993; 128(1):79-88. [PubMed: 8418785]

8. Bulletines ACoP. ACOG Practice Bulletin No. 74. Antibiotic prophylaxis for gynecologic procedures. Obstetrics and Gynecology. 2006; 108(1):225-234. [PubMed: 16816087]

9. Rodrigues AS,ML. Incidence of surgical site infection with pre-operative skin preparation using 10\% polyvidone-iodine and 0.5\% chlorhexidine-alcohol. Rev Col Bras Cir. 2013; 40(6):443-448. [PubMed: 24573620]

10. Prevention CfDCa. , editor. Hospital Infections Program NCfID, Centers for Disease Control and Prevention, Public Health Service, U.S. Department of Health and Human Services. Guideline for Prevention of Surgical Site Infection. 1999. 1999 
11. Eaglstein WD,SC, Mehle AL, Mertz PM. Optimal Use of an Occlusive Dressing to Enhance Healing Effect of Delayed Application and Early Removal on Wound Healing. Archives of Dermatology. 1988; 124:392-395. [PubMed: 3345089]

12. RA G. Prevention of intraoperative wound contamination with chlorhexidine shower and scrub. J Hosp Infect. 1988; 11(Supplement B):5-9. [PubMed: 2898503]

13. Keenan JE, Speicher PJ, Thacker JM, Walter M, Kuchibhatla M, Mantyh CR. The preventive surgical site infection bundle in colorectal surgery: An effective approach to surgical site infection reduction and health care cost savings. JAMA Surgery. 2014; 149(10):1045-1052. [PubMed: 25163027]

14. Cima RD,E, Lovely J, Pendlimari R, Aronhalt K, Nehring S, Hyke R, Tyndale D, Rogers J, Quast L. Colorectal surgery surgical site infection reduction program: a national surgical quality improvement program-driven multidisciplinary single-institution experience. Journal of the American College of Surgeons. 2013; 216(1):23-33. [PubMed: 23127793]

15. Novetsky AZIG,SR, Ioffe YJ, Kizer NT, Hagemann AR, Powell MA, Thaker PH, Mutch DG, Massad LS. A phase II trial of a surgical protocol to decrease the incidence of wound complications in obese gynecologic oncology patients. Gynecologic Oncology. 2014; 134(2):233237. [PubMed: 24952366]

16. Johnson MP, Kim SJ, Langstraat CL, et al. Using Bundled Interventions to Reduce Surgical Site Infection After Major Gynecologic Cancer Surgery. Obstetrics \& Gynecology. 2016; 127(6):11351144. [PubMed: 27159744]

17. University Health System Consortium Clinical Database/Resource Manager. 2013. https:// www.vizientinc.com/. Accessed 04/06/2016

18. American College of Surgery National Surgical Quality Improvement Program SCR Operations Manual. 2013. http://www.cdc.gov/nhsn/CPTcodes/ssicpt.html

19. Mangram AJ, Horan TC, Pearson ML, Silver LC, Jarvis WR. Guideline for Prevention of Surgical Site Infection, 1999. American Journal of Infection Control. 1999; 27(2):97-134. [PubMed: 10196487]

20. Nelson Richard LGE, Barbateskovic Marija. Antimicrobial prophylaxis for colorectal surgery. Cochrane Database of Systematic Reviews. 2014; 5

21. Veiga DD,CA, Veiga-Filho J, Figueiras RG, Vieira RB, Garcia ES, Silva VV, Novo NF, Ferreira LM. Randomized controlled trial of the effectiveness of chlorhexidine showers before elective plastic surgical procedures. Infect Control Hosp Epidemiol. 2009; 30(1):77-79. [PubMed: 19046051]

22. Bratzler DWDE, Olsen KM, et al. Clinical practice guidelines for antimicrobial prophylaxis in surgery. Am J Health Syst Pharm. 2013; 70(3):195-283. [PubMed: 23327981]

23. The University of Texas Publically Available Salary Information Compiled by the Texas Tribune. 2015. http://salaries.texastribune.org/the-university-of-texas-md-anderson-cancer-center/ departments/gyn-onc-reproductive-med/positions/coord-research-data. Accessed 2/4/16, 2016

24. Singh AB,SM, Muder RR, Lee BY. An economic model: value of antimicrobial-coated sutures to society, hospitals, and third-party payers in preventing abdominal surgical site infections. Infect Control Hosp Epidemiol. 2014; 35(8):1013-1020. [PubMed: 25026618]

25. Gheorghe AR,TE, Pinkney TD, Bartlett DC, Morton D, Calvert M. The cost-effectiveness of wound-edge protection devices compared to standard care in reducing surgical site infection after laparotomy: an economic evaluation alongside the ROSSINI trial. Public Library of Science. 2014; 18(9)

26. Anthony TM,BW, Sum-Ping JT, Lenkovsky F, Vornik VD, Parker BJ, McFarlin JE, Hartless K, Huerta S. Evaluating an evidence-based bundle for preventing surgical site infection: a randomized trial. Archives of Surgery. 2011; 146(3):263-269. [PubMed: 21079110]

27. Al-Niaimi AN, Ahmed M, Burish N, et al. Intensive postoperative glucose control reduces the surgical site infection rates in gynecologic oncology patients. Gynecologic Oncology. 2015; 136(1):71-76. [PubMed: 25263249] 


\section{SYNOPSIS}

A bundled intervention successfully reduced surgical site infections following surgery for gynecologic malignancy and benign disease. The intervention resulted in significant costsavings from a hospital perspective and reduced patient morbidity. 


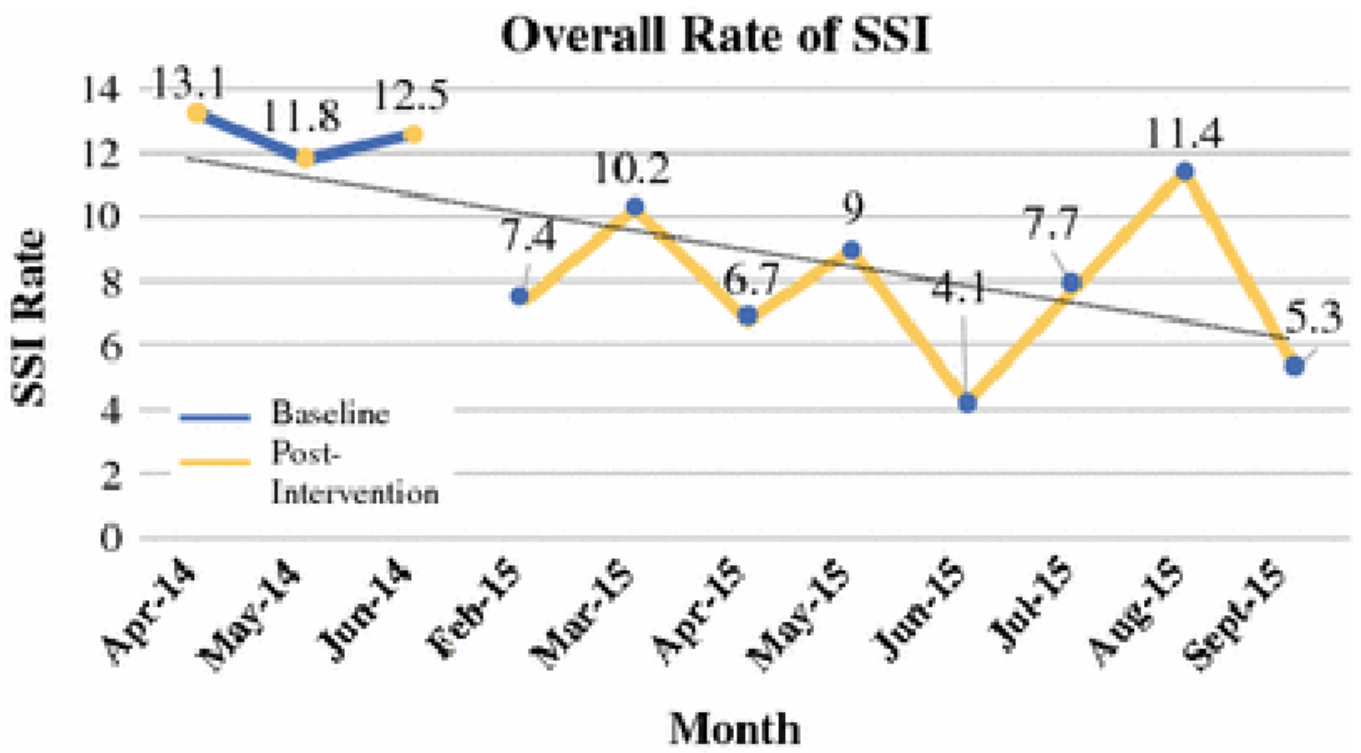

Figure 1.

Overall Rate of SSI 
Table 1

Patient Demographics

\begin{tabular}{|c|c|c|c|}
\hline Characteristic & Baseline N=232 (\%) & Post-Intervention $\mathrm{N}=555(\%)$ & P-value \\
\hline Median Age & 57 & 57 & 0.17 \\
\hline Median BMI & 28.9 & 28.4 & 0.30 \\
\hline Pre-operative Chemotherapy & $40(17)$ & $75(14)$ & 0.19 \\
\hline Pre-operative Radiation & $5(2)$ & $8(1)$ & 0.54 \\
\hline Current Smokers & $19(8)$ & $45(8)$ & 0.99 \\
\hline Diabetic & $36(16)$ & $62(11)$ & 0.10 \\
\hline Race/Ethnicity & & & 0.61 \\
\hline White & $158(68)$ & $388(70)$ & \\
\hline Hispanic & $30(13)$ & $78(14)$ & \\
\hline Black & $26(11)$ & $58(10)$ & \\
\hline Asian & $17(7)$ & $27(5)$ & \\
\hline Other/Unknown & $1(<1)$ & $4(1)$ & \\
\hline Indication for Surgery & & & 0.01 \\
\hline Benign Disease & $55(24)$ & $180(32)$ & \\
\hline Malignancy & $177(76)$ & $375(68)$ & \\
\hline Cancer Type & & & 0.28 \\
\hline Ovary/Peritoneal & $97(42)$ & $173(31)$ & \\
\hline Uterine & $60(26)$ & $144(26)$ & \\
\hline Cervix & $15(6)$ & $46(8)$ & \\
\hline Ovarian \& Endometrial & $3(1)$ & $4(1)$ & \\
\hline Other & $2(1)$ & $8(2)$ & \\
\hline Median EBL (mL) & 100 & 50 & 0.006 \\
\hline Median Surgical Time (hours) & 2.67 & 2.62 & 0.30 \\
\hline Appropriate Antibiotic Prophylaxis & $152(66)$ & $409(74)$ & 0.03 \\
\hline Appropriate Antibiotic Re-dosing & $208(90)$ & $524(94)$ & 0.02 \\
\hline Surgical Approach & & & 0.06 \\
\hline Laparotomy & $112(48)$ & $227(41)$ & \\
\hline Minimally Invasive & $120(52)$ & $328(59)$ & \\
\hline Bowel Surgery & $54(23)$ & $114(21)$ & 0.39 \\
\hline
\end{tabular}




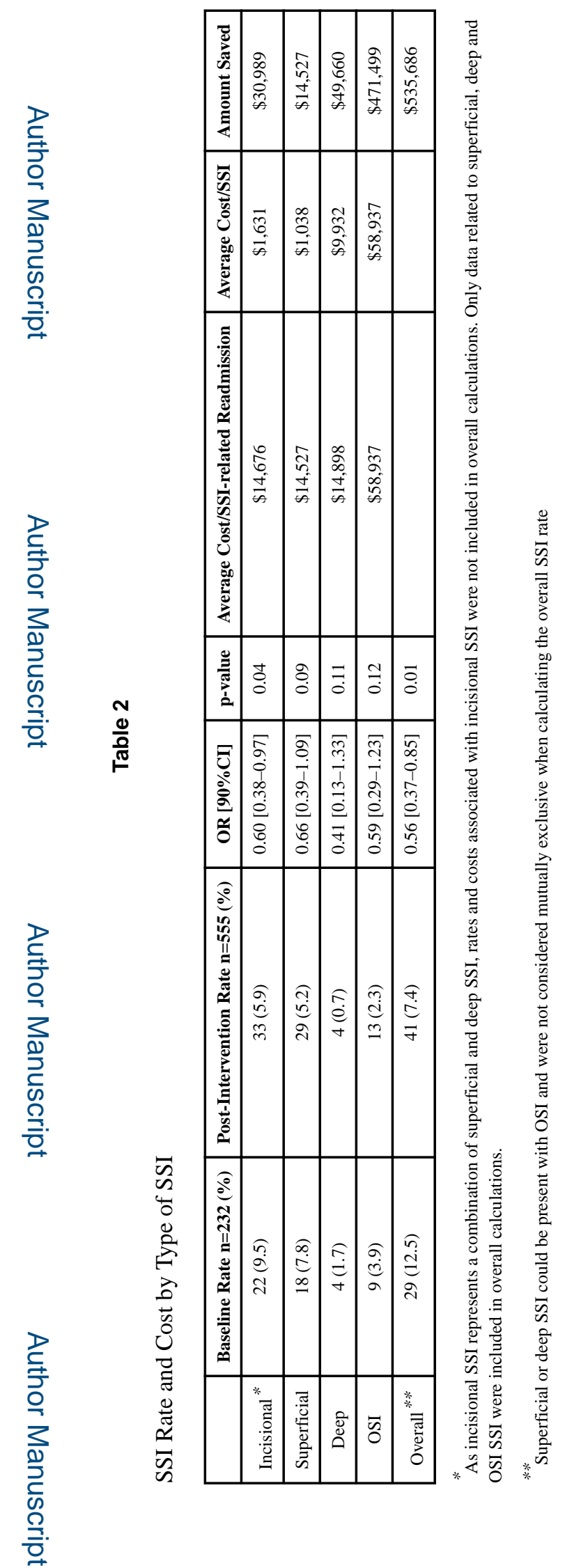

Ann Surg Oncol. Author manuscript; available in PMC 2018 February 01. 


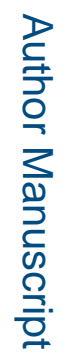



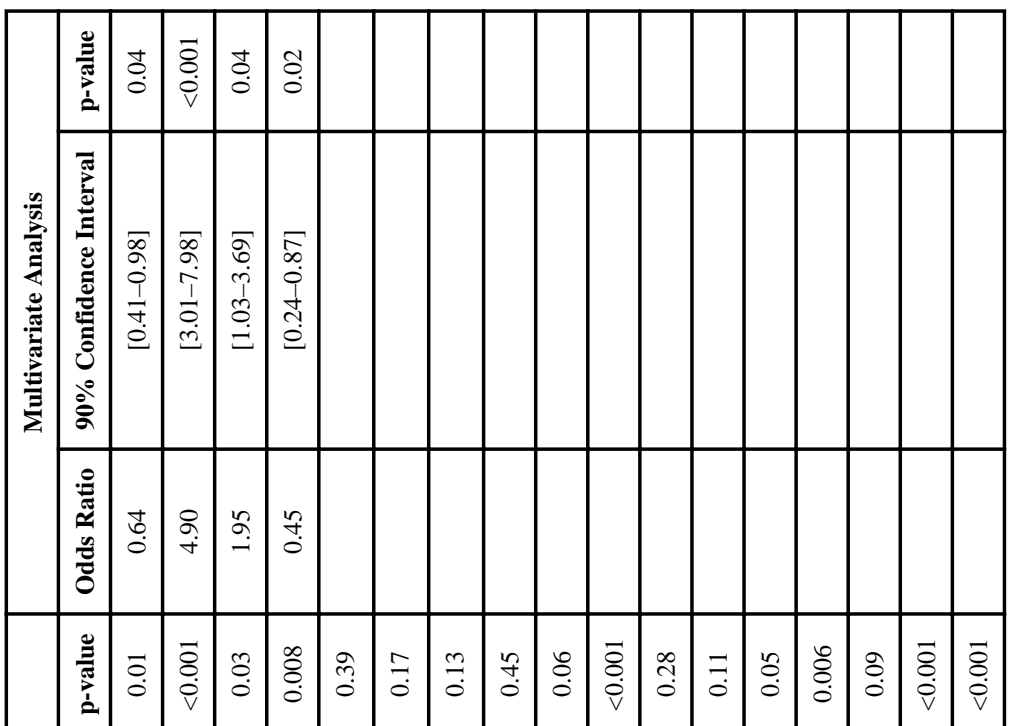

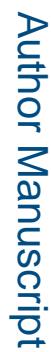

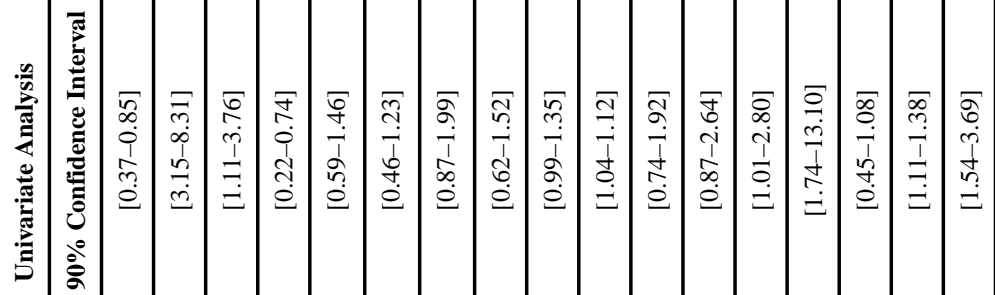

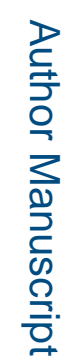

\begin{tabular}{|c|c|c|c|c|c|c|c|c|c|c|c|c|c|c|c|c|}
\hline 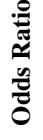 & $\begin{array}{l}0 \\
\stackrel{0}{0} \\
0\end{array}$ & $\vec{z}$ & 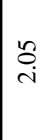 & $\begin{array}{l}q \\
0 \\
0\end{array}$ & O̊. & $\begin{array}{l}n \\
\\
0\end{array}$ & लै & $\begin{array}{l}\hat{a} \\
0\end{array}$ & $\stackrel{\circ}{=}$ & $\stackrel{\infty}{\stackrel{\leftrightarrow}{-}}$ & $\stackrel{9}{=}$ & & & 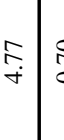 & \begin{tabular}{l|l}
$R$ & \multirow{O}{0}{} \\
.
\end{tabular} & ले \\
\hline 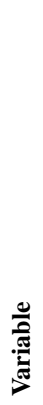 & 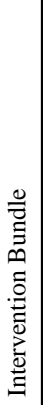 & 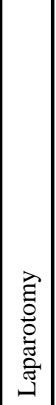 & 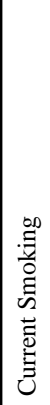 & 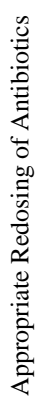 & 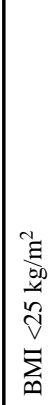 & 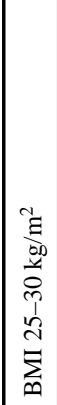 & 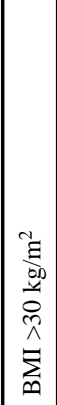 & 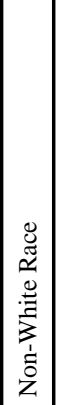 & 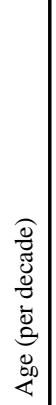 & 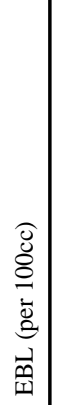 & 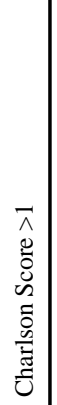 & 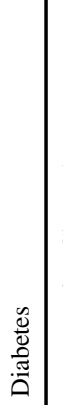 & 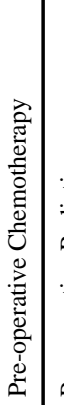 & 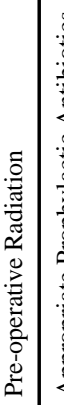 & 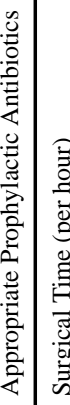 & (2) \\
\hline
\end{tabular}

Ann Surg Oncol. Author manuscript; available in PMC 2018 February 01. 
Table 4

Cost of the Intervention Bundle

\begin{tabular}{|l|c|}
\hline & Cost \\
\hline Antibacterial Soap & $\$ 0.34$ per surgery \\
\hline Patient Education Handouts & $\$ 0$ (incorporated in existing handouts) \\
\hline Change of Electrocautery Device & $\$ 2.78$ per open surgery \\
\hline Change of Gloves & $\$ 3.88$ per surgery \\
\hline Change of Suction tip & $\$ 0.40$ per open surgery \\
\hline Change of Gown & $\$ 2.52 \times$ One person/surgery $\times 63 \%$ of surgeries \\
\hline Separate instruments & $\$ 878.23$ per tray $(\mathrm{x} 6$ trays) \\
\hline Cleaning separate instruments & $\$ 6.50$ per open surgery \\
\hline Post-discharge Phone Call & $\$ 0$ (incorporated into existing process) \\
\hline Total per intervention & $\$ 19.26$ \\
\hline Total Cost During Study Period & $\$ 10,689.96(\$ 1,336.25 /$ month) \\
\hline
\end{tabular}

